\title{
Arte parietal paleolítico en la cueva de Aitzbitarte V (Errenteria, Gipuzkoa): un nuevo conjunto diacrónico en la Región Cantábrica oriental ${ }^{*}$
}

\author{
Paleolithic parietal art in the Aitzbitarte V Cave (Errenteria, Gipuzkoa): a new diachronic \\ set in the eastern Cantabrian Region
}

\author{
Diego Garate ${ }^{\mathrm{a}}$, Olivia Rivero ${ }^{\mathrm{b}}$, Joseba Rios-Garaizar', Iñaki Intxaurbe ${ }^{\mathrm{d}}$ y Sergio Salazar ${ }^{\mathrm{b}}$
}

\begin{abstract}
RESUMEN
El arte parietal paleolítico de la cueva de Aitzbitarte V se descubre en 2015, en el marco de las labores de prospección desarrolladas en la última década en el oriente cantábrico. Hemos documentado una decena de grabados situados en tres sectores profundos de la cavidad y compuestos por representaciones de bisontes y líneas. Las características formales de los primeros son específicas del arte gravetiense en el caso de los sectores A, C y D, y del Magdaleniense en el sector B. En ambos casos las convenciones gráficas presentan paralelos continentales, con el S/SO francés en el primer caso, y con los conjuntos pirenaicos en el segundo. De esta manera, en la cueva de Aitzbitarte V se identifican dos fases decorativas correspondientes a dos periodos distantes en el tiempo, siendo el primer caso de recurrencia gráfica reconocido en la Región Cantábrica oriental.
\end{abstract}

teristics of the bisons are specific to Gravettian art in the case of sectors $A, C$ and $D$, and to the Magdalenian in sector B. In both cases, the graphic conventions have parallels in the French record, with the French $S / S W$ in the first case, and with the Pyrenean groups in the second. Thus, in the cave of Aitzbitarte V two decorative phases are identified, corresponding to two chronologically distant events. This is the first case of graphic recurrence recognized in the eastern Cantabrian Region.

Palabras clave: Aitzbitarte V; Gravetiense; Magdaleniense; Arte rupestre; Grabado; Simbolismo; Región Cantábrica; Documentación tridimensional; Bisonte.

Key words: Aitzbitarte V; Gravettian; Magdalenian; Rockart; Symbolism; Cantabrian region; Engraving; Three-dimensional documentation; Bison.

\begin{abstract}
The Paleolithic parietal art of the Aitzbitarte $V$ Cave was discovered in 2015, during the surveying work carried out in the east of Cantabrian Region. We have documented ten engravings located in three deep sectors of the cave. The engravings represent bisons and lines. The formal charac-
\end{abstract}

\section{INTRODUCCIÓN}

En el presente trabajo damos a conocer las figuras grabadas de cronología paleolítica de la cueva de Aitzbitarte V (Errenteria, Gipuzkoa). La aportación de este conjunto inédito resulta de especial interés para abor-

* El estudio forma parte de los proyectos de investigación "Before art: social investment in symbolic expressions during the Upper Palaeolithic (B-Art)" (PID2019-107262GB-I00 IP Diego Garate), financiado por el Ministerio de Ciencia, Innovación y Universidades, y "Learning and development of artistic abilities in Anatomically Modern Humans; a multidisciplinary approach (ApArt)" (HAR2017-87739-P IP Olivia Rivero), financiado también por el Ministerio de Ciencia, Innovación y Universidades. I. Intxaurbe es beneficiario de una beca predoctoral (PIF 2019) de la Universidad del País Vasco (UPV/EHU). La investigación contó también con apoyo económico de la Diputación Foral de Gipuzkoa y el municipio de Errenteria.

a Instituto Internacional de Investigaciones Prehistóricas de Cantabria (IIIPC, Gobierno de Cantabria, Universidad de Cantabria, Santander). Edificio Interfacultativo, Avda. Los Castros s/n. 39005 Santander. España. Correo e.: diego.garate@unican.es https://orcid.org/0000-0001-6685-9588

b Dpto. de Prehistoria, Historia Antigua y Arqueología. Universidad de Salamanca. Cervantes s/n. 37002 Salamanca. España.

Correo e.: oliviariver@usal.es https://orcid.org/0000-0002-0730-4854; SS: correo e.: salazar.laredo@gmail.com https://orcid.org/0000-0002-2892-3945

c Centro Nacional de Investigación sobre la Evolución Humana (CENIEH). Paseo Sierra de Atapuerca s/n. 09002 Burgos. España. Correo e.: joseba.rios@cenieh.es https://orcid.org/0000-0001-8474-2156

d Dpto. de Geología. Euskal Herriko Unibertsitatea/Universidad del País Vasco. 48940 Leioa. España. Correo e.: inaki.intxaurbe@ehu.eus https://orcid.org/0000-0003-3643-3177

Recibido 21-V-2020; aceptado 11-VIII-2020.

Copyright: (C) 2020 CSIC. Este es un artículo de acceso abierto distribuido bajo los términos de la licencia de uso y distribución "Creative Commons Reconocimiento 4.0 Internacional” (CC BY 4.0) 
dar el desarrollo del arte parietal paleolítico por dos razones fundamentales: por un lado, el establecimiento de similitudes gráficas en diversas áreas geográficas a través de las convenciones gráficas presentes en diversos periodos (Fortea et al. 2004; Feruglio et al. 2011) y, por otro, para abordar la recurrencia gráfica en una misma cavidad durante diversos periodos (González-Sainz 2004; Fortea 2007).

Durante el transcurso de las prospecciones arqueológicas para la identificación de grafías parietales paleolíticas en el Cantábrico oriental, D. Garate y J. Rios-Garaizar, junto con J. Busselo, G. Studer, S. Orbegozo e I. Errazkin, miembros del Félix Ugarte Espeleologia Elkartea, descubren en septiembre de 2015 las primeras figuras de bisontes grabados en Aitzbitarte V. Una semana después J. Busselo y G. Studer localizan más representaciones grabadas en las cuevas de Aizbitarte III y IX (Garate et al. 2016).

El hallazgo se enmarca en la notable reactivación de las investigaciones sobre arte parietal paleolítico en el norte de la península ibérica, especialmente en el sector oriental de la Cordillera Cantábrica, producida durante la última década. Tradicionalmente, este sector ha tenido una densidad de cuevas decoradas sensiblemente menor que en las regiones limítrofes -Región Cantábrica centro-occidental, Pirineos y Périgord- a pesar de su posición geoestratégica en el punto central de conexión entre estas, y de contacto con la península ibérica y el resto del continente europeo.

Las nuevas investigaciones han triplicado la cantidad de conjuntos parietales conocidos (Fig. 1), pasan- do de una decena escasa a más de una treintena, incluyendo grandes santuarios magdalenienses como Atxurra y Armintxe. Además 14 de esas nuevas cuevas, una cantidad considerable, se decoraron con anterioridad al Magdaleniense, en periodos desconocidos en este área hasta el momento (Garate 2018).

\section{LOCALIZACIÓN, DESCRIPCIÓN Y OCUPACIÓN HUMANA DE LA CAVIDAD}

La colina de Aitzbitarte presenta una fuerte actividad kárstica, con una treintena de pequeñas oquedades, simas y cuevas documentadas hasta el momento (Manteca et al. 1997). Las entradas de las cuevas con yacimiento arqueológico se localizan en la ladera oeste, a escasos $40 \mathrm{~m}$ de altura sobre el cauce actual del arroyo que discurre a sus pies, un afluente del río Urumea que actualmente desemboca en Donostia/San Sebastián a unos $12 \mathrm{~km}$ de distancia en línea recta. La cuenca del Urumea se sitúa en el extremo oriental de la Cornisa Cantábrica, muy cerca de las estribaciones pirenaicas y a escasa distancia de la frontera actual entre España y Francia, es decir, entre la península ibérica y el resto del continente europeo. El proceso de karstificación se desarrolla en las calcarenitas bioclásticas del Albiense Superior. Allí el descenso del nivel freático y la variación en el aporte sedimentario a las cavidades han propiciado la formación de galerías horizontales mediante la paragénesis y un sistema de cavidades multinivel (Fig. 2.2).

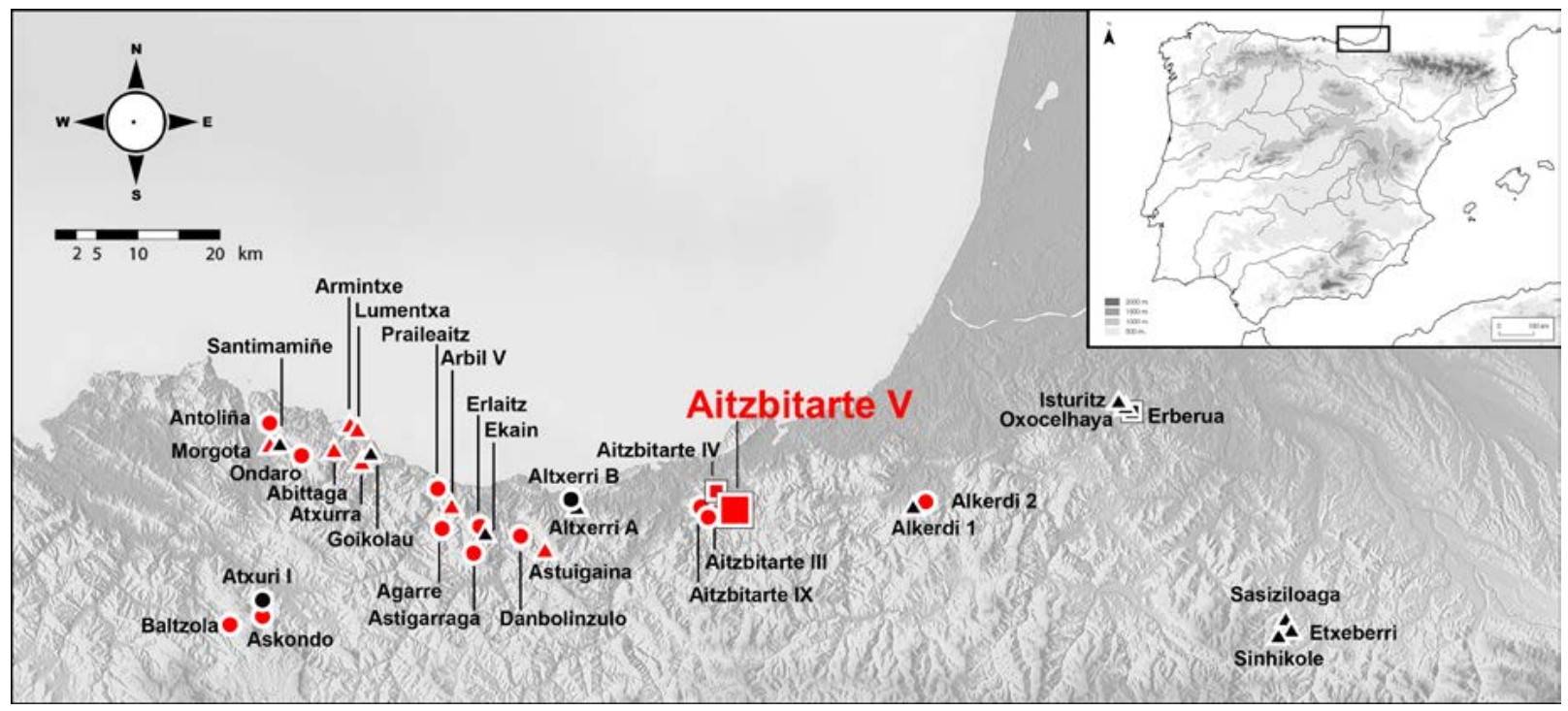

Fig. 1. Cuevas decoradas en el Cantábrico oriental y los Pirineos occidentales (mapa base: https://maps-for-free.com): negro descubierto durante el siglo XX; rojo descubierto en el siglo XXI; círculo para conjuntos premagdalenienses; triángulo para los magdalenienses; cuadrado para conjuntos complejos (premagdalenienses y magdalenienses). En color en la edición electrónica. 
La cueva V (Fig. 2.1) se sitúa unos $20 \mathrm{~m}$ por encima de las cavidades arqueológicas III, IV y IX, en la misma ladera occidental de la colina, pero su desarrollo es sensiblemente menor: $60 \mathrm{~m}$ desde la entrada hasta la sala final (Fig. 2.3). Originalmente la cueva $\mathrm{V}$ se describió como un único conducto meandriforme de unos $25 \mathrm{~m}$ de desarrollo, unos $3 \mathrm{~m}$ de anchura y unos 2,20 de altura (Altuna et al. 1995), en cuyo fon- do se observaba lo que parecían unas gateras obstruidas. Las distintas topografías realizadas dentro del sistema kárstico de Aitzbitarte mostraban que estas colmataciones quedaban a escasa distancia del final de la conocida como "Galería de los Osos", a la que se accedía desde la cueva IV a través de un conducto vertical ascendente de unos $25 \mathrm{~m}$.
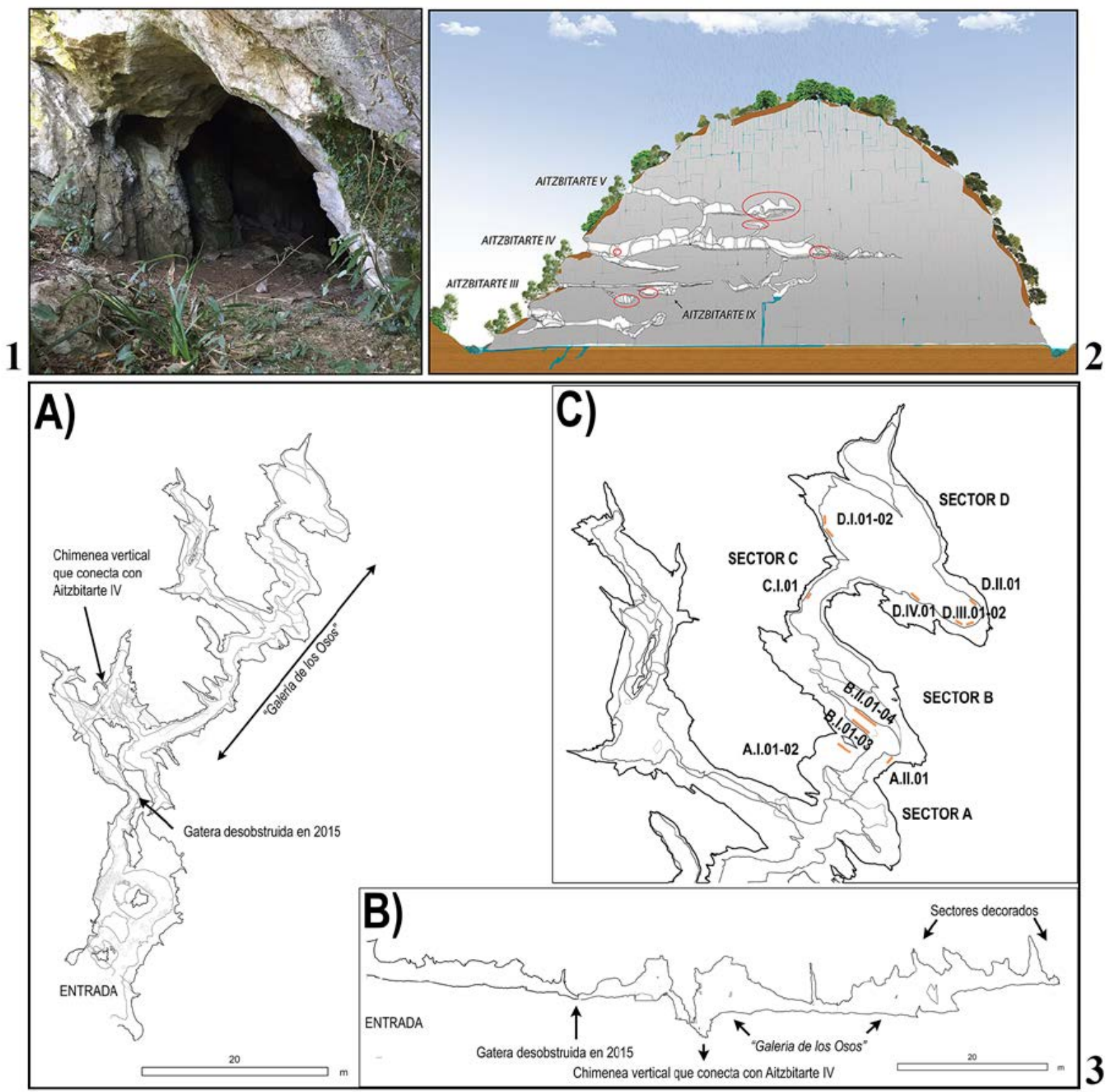

Fig. 2. Localización de la cueva Aitzbitarte V en la colina: 1. Entrada de la cavidad (D. Garate), 2. Corte vertical de la colina con los principales fenómenos espeleológicos indicados y los sectores decorados señalados con círculo (a partir del grupo de espeleología "Felix Ugarte Elkartea"), 3. Topografía de la cueva indicando las áreas decoradas (a partir de Gim-Geomatics, S.L.): A) planta, B) alzado, C) planta en detalle del sector decorado indicando las grafías encontradas. En color en la edición electrónica. 
En 2015, las tareas de desobstrucción a cargo del grupo de espeleología "Felix Ugarte Elkartea" revelaron que efectivamente este conducto colmatado conectaba con la parte superior de la chimenea vertical que unía la cueva IV con la "Galería de los Osos" (Fig. 2.3). También se pudo observar que la primera sala a la derecha de la "Galería de los Osos" conectaba mediante un conducto corto, actualmente colmatado, con la zona de la entrada de la cueva V. Dicha galería debe su nombre a la multitud de oseras que se conservan en el suelo de la cueva, probablemente atribuibles a la especie Ursus spelaeus extinta hace unos 26-24.000 años (Baca et al. 2016). Estos osos además marcaron las paredes de la cueva con numerosos zarpazos. Aparecen incluso en los paneles que contienen los grabados paleolíticos, los cuales siempre se les superponen. Todas estas evidencias sugieren que los conductos principales de la cavidad apenas han sufrido aportes sedimentarios o erosiones importantes en los últimos 25.000 años. Las dimensiones mínimas del primer tramo de la galería después del pozo son $2 \mathrm{~m}$ de anchura y $3 \mathrm{~m}$ de altura. En los laterales del conducto principal se abren multitud de conductos y grietas, algunas totalmente colmatadas. Tras pasar este conducto y superar un pequeño obstáculo vertical se accede al tramo final de la galería, donde se sitúan los tres sectores decorados de la cueva. Todas las paredes de esta zona, incluyendo las superficies decoradas, están alteradas por numerosos grafitis y en los suelos hay acumulaciones recientes de carbones, debido a visitas incontroladas desde la chimenea que conecta con Aitzbitarte IV.

La ocupación humana de la colina de Aitzbitarte se prolonga a lo largo de todo el Paleolítico Superior de manera complementaria en las cavidades excavadas hasta día de hoy. El erudito local Conde de Lerchundi inicia las intervenciones arqueológicas en 1892 en la cueva IV, que será además visitada por importantes prehistoriadores del momento como H. Breuil, É. Harlé, H. Obermaier y J. Bouyssonie. Las primeras excavaciones científicas se desarrollan entre 1960 y 1964 (Barandiaran et al. 1965) y en esa misma época se descubrirán también algunas evidencias arqueológicas en la cueva V (Altuna 2004). Entre 1985 y 2002 J. Altuna excava en el yacimiento de la cavidad III (Altuna et al. 2011, 2017). En 2012 D. Garate y J. RiosGaraizar (Garate et al. 2013) descubren las primeras evidencias de arte parietal paleolítico de las cuevas de Aitzbitarte en la IV.

En la cueva de Aitzbitarte, en 1961, V. A. Laburu, J. Louvelli e I. Sánchez, colaboradores de las excavaciones realizadas por J. M. Barandiarán en la década de los 60 en la cueva IV, dan noticia del hallazgo de restos arqueológicos en su entrada. Entre ellos hay industria lítica, cerámica, macrofauna, moluscos y un fragmento de cráneo humano (Altuna et al. 1995). Ya en 2015, en el marco de la desobstrucción ejecutada por el grupo espeleológico "Felix Ugarte Elkartea", origen del descubrimiento de arte rupestre en Aitzbitarte $\mathrm{V}$, se han localizado abundantes fragmentos cerámicos prehistóricos, algunos probablemente de la Edad de Bronce, así como una lámina de sílex de gran tamaño obtenida mediante presión. Es decir, hasta la actualidad no se ha desarrollado una investigación sistemática sobre su depósito sedimentario.

\section{ESTUDIO DEL DISPOSITIVO GRÁFICO PARIETAL}

Los motivos grabados en la cueva de Aitzbitarte V se concentran en el tramo final de la cavidad, que comienza a unos $50 \mathrm{~m}$ de la entrada y que tiene unos $25 \mathrm{~m}$ de desarrollo (Fig. 2). Las grafías se agrupan en cuatro sectores con grabados de cronología paleolítica, tres de ellos atribuidos al Gravetiense $-\mathrm{A}, \mathrm{C}$ y $\mathrm{D}-\mathrm{y}$ otro al Magdaleniense Medio -B-.

\subsection{Metodología de documentación}

Los resultados obtenidos en Aitzbitarte V son el producto de un proceso exhaustivo de prospección y documentación, realizado tras descubrirse los primeros grabados en el sector B. Para ello se adaptó la metodología de Sanchidrián y Medina-Alcaide (2017) a los condicionantes específicos de la cavidad y de sus grabados. Durante el siglo XX las visitas incontroladas, ya mencionadas, cubrieron de grafitis y de frotados todas las paredes. Como consecuencia de dichas acciones vandálicas no descartamos la pérdida de grafías paleolíticas, cuyo precario estado de conservación ha dificultado el estudio. Ademas, los zarpazos de osos de las cavernas que recubren las paredes también dificultan las tareas de prospección.

La prospección sistemática de las paredes y de los techos implicó el reconocimiento detenido de todas las superficies rocosas accesibles directamente o mediante material espeleológico de progresión. Los motivos grabados se definieron por observación directa, con lupa o con microscopio digital. Los datos se registraron en fichas a nivel de grafía, panel y sector. La documentación fotográfica y restitución infográfica de los motivos siguió el protocolo ya establecido (Rivero et al. 2019), usando la fotografía digital convencional y la fotogrametría de objeto cercano para la obtención de modelos tridimensionales. Para la restitución gráfica recurrimos al tratamiento digital y a la infografía de las imágenes empleando dos tabletas gráficas profesionales. Las restituciones gráficas se corrigieron in situ directamente en 
la versión digital. La documentación y registro del espacio decorado se obtuvo mediante un escaneo tridimensional del conjunto de la cavidad.

\subsection{Descripción del dispositivo}

El dispositivo gráfico de la cueva de Aitzbitarte V se divide en cuatro sectores distintos, todos ellos situados en su tramo final.

\subsubsection{Sector $A$}

Este primer sector decorado se localiza justo antes del recodo a izquierda que conduce a la sala final. Ascendiendo lateralmente por una banqueta inclinada se accede a una estrecha repisa a $3 \mathrm{~m}$ de altura que actúa como balcón al pasillo contiguo. Allí se sitúa el siguiente sector con dos representaciones parciales de bisonte (cabeza, cuernos y arranque de la giba) A.I.1 y A.II.1 y una serie de líneas no figurativas A.I.2 (Fig. 3).
El panel A.I está cerca de la repisa, contiguo al balcón, sobre una superficie cubierta por zarpazos de oso:

A.I.1- Cabeza de bisonte orientada a la izquierda compuesta por la línea frontal unida a un cuerno, una serie de trazos cortos representando la testuz, el segundo cuerno y una serie de incisiones cortas e inclinadas a modo de giba. Las dimensiones máximas son de 19 × $9 \mathrm{~cm}$. Está a $133 \mathrm{~cm}$ de la repisa.

A.I.2- Signo compuesto por dos líneas curvas convergentes en forma conopial. Las dimensiones máximas son de 23 x $13 \mathrm{~cm}$. Se sitúa a $135 \mathrm{~cm}$ de la repisa.

El panel A.II se localiza al otro lado del balcón, sobre la pared de la propia cueva, accesible desde el fondo de la repisa. También son numerosos los zarpazos de oso.

A.II.1- Cabeza de bisonte orientada a la izquierda formada por la línea frontal unida a un cuerno, una serie de trazos ligeramente inclinados representando la testuz y otros menos alineados para la barba, el segundo cuerno y un único trazo indicando el arranque de la giba. A derecha de la grafía se observan 4 zarpazos de oso profundamente incisos. Las dimensiones máximas son de $12 \times 14 \mathrm{~m}$. Está a $60 \mathrm{~cm}$ de la repisa.
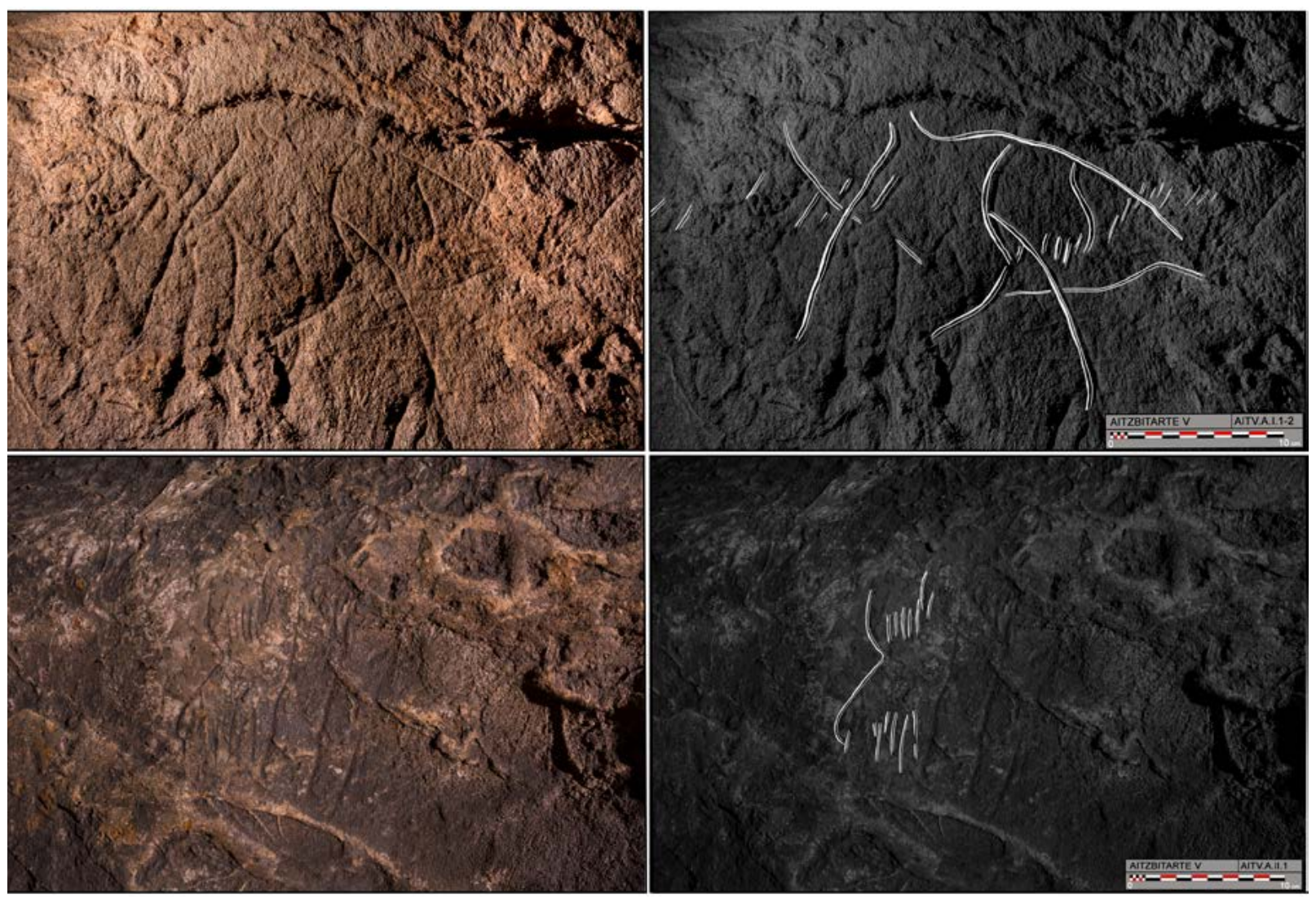

Fig. 3. Fotografía y calco de los bisontes A.I.1 y A.II.2 y del signo A.I.2, grabados en el sector A de Aitzbitarte V (O. Rivero y D. Garate). En color en la edición electrónica. 


\subsubsection{Sector $B$}

Un estrecho paso en forma de arco, a la altura del suelo, permite situarse al otro lado de la repisa y del balcón. En este estrecho pasillo de unos $3 \mathrm{~m}$ de desarrollo y $1 \mathrm{~m}$ de anchura, se han grabado cuatro bison- tes más o menos completos, situados dos a dos en cada lado del pasillo.

En el panel izquierdo B.I según se accede al sector, se observan dos bisontes enfrentados de más o menos $1,25 \mathrm{~m}$ de longitud y una línea curva (Fig. 4).
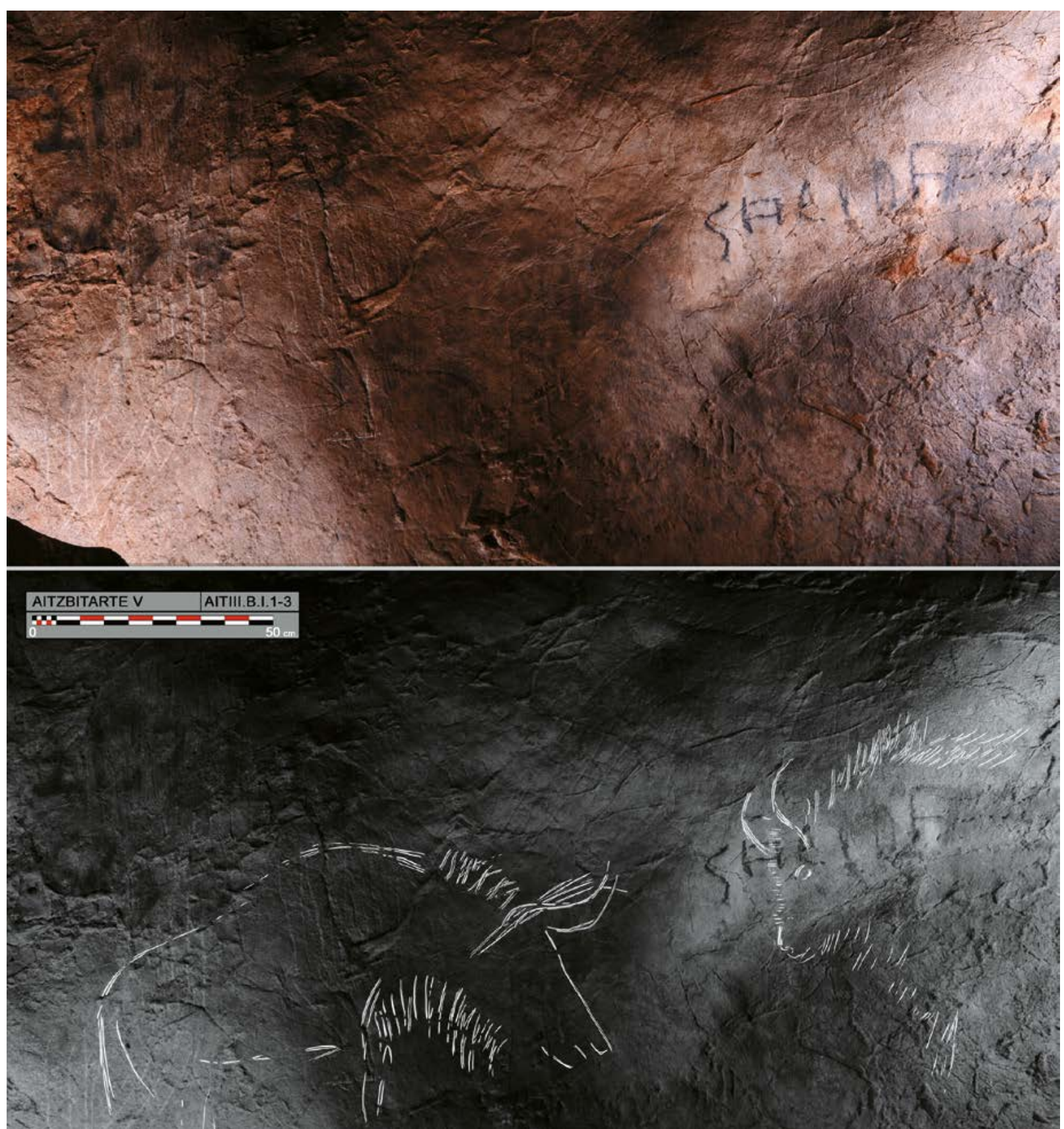

Fig. 4. Fotografía y calco del panel B.I con los bisontes B.I.1 y B.I.3 y la línea curva B.I.2, grabados en el sector B de Aitzbitarte V (O. Rivero y D. Garate). En color en la edición electrónica. 
B.I.1- Justo encima del arco de acceso y aprovechando una convexidad se localiza el primer bisonte, orientado a derecha y muy detallado (Fig. 5). Se observan los cuernos sinuosos en "S" y con doble línea, la frontonasal rectilínea, la giba con el pelaje indicado mediante trazos cortos verticales, el lomo y apoyándose sobre el límite del arco el tren trasero con la cola. La barba, la pata delantera y el vientre se reconocen con más dificul- tad al estar parcialmente borrados. Bajo su lomo se observan zarpazos de oso y un tizonazo negro se superpone a la cabeza. La parte inferior del animal está deteriorada por un frotado recurrente al servir de apoyo para cruzar el arco de acceso. Las dimensiones máximas son de 108 x 59 cm. Está a $157 \mathrm{~cm}$ del suelo (Fig. 5).

B.I.2- Línea sinuosa compuesta por múltiples trazos situada bajo los cuernos del bisonte anterior. Las
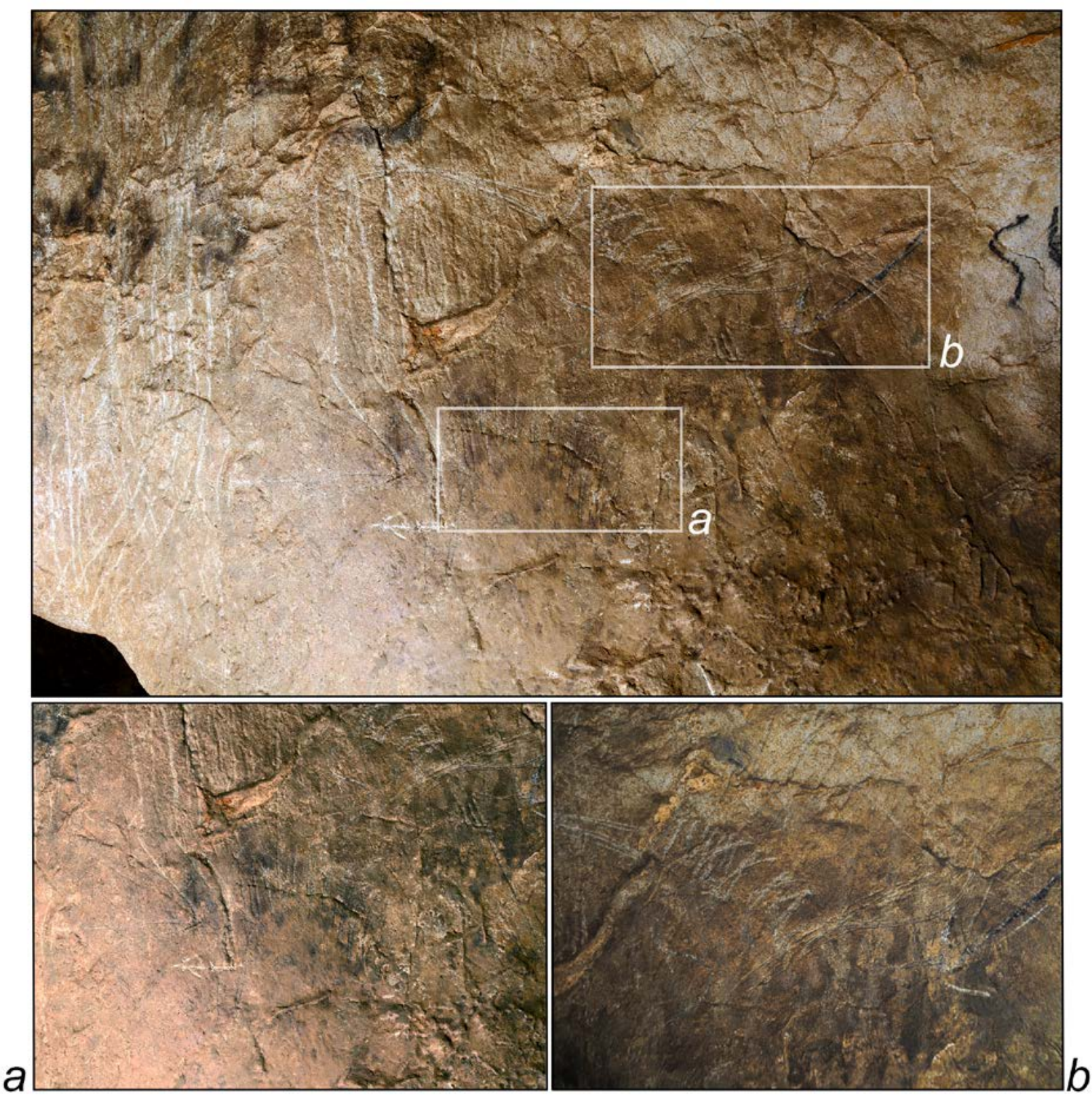

Fig. 5. Fotografía general y de detalle del bisonte B.I.1, grabado en el sector B de Aitzbitarte V (O. Rivero y D. Garate). En color en la edición electrónica.

Trab. Prehist., 77, N. ${ }^{\circ}$ 2, julio-diciembre 2020, pp. 320-336, ISSN: 0082-5638

https://doi.org/10.3989/tp.2020.12259 
dimensiones máximas son de 18 x $5 \mathrm{~cm}$ y se sitúa a $160 \mathrm{~cm}$ del suelo.

B.I.3- A continuación de la grafía anterior pero enfrentada con ella, se observa otro bisonte parcial (Fig. 6). Tiene dos cuernos en perspectiva hechos con trazo múltiple, unos trazos cortos perpendiculares anteceden a la línea frontal, que a su vez finaliza en una boca bien perfilada y con el pelaje de la barba abundante. La giba se representa mediante trazos entrecruzados parece que sin continuación en el lomo ni en el tren trasero. La parte inferior está afectada por el frotamiento de las arcillas depositadas sobre la roca soporte del pasadizo y sobre la cabeza y los cuernos se lee "SALIDA" escrito en carbón. Las dimensiones máximas son de $70 \times 78 \mathrm{~cm}$. Está a $162 \mathrm{~cm}$ del suelo (Fig. 6).

En la pared opuesta B.II se localiza otro panel con grabados. La lectura es más complicada ya que los surcos son más finos y el grado de deterioro es todavía mayor, debido a grafitis, aportes recientes de arcilla y rozamientos. Se identifican dos figuras de bisonte (Fig. 7) y una línea grabadas, además de una pequeña mancha en rojo.

B.II.1- Cabeza orientada a la izquierda. Se observa con cierta dificultad por la superposición de grafitis. Tiene ambos cuernos en perspectiva, dos trazos curvos conformando un ojo, una serie de trazos largos inclinados para la giba y otra serie más corta para la línea frontal. La boca y la barba se han ejecutado mediante incisiones paralelas verticales. Las dimensiones máximas son de 31 x $98 \mathrm{~cm}$. Se sitúa a $162 \mathrm{~cm}$ del suelo.

B.II.2- Bisonte orientado a la derecha y afrontado al anterior. Se distinguen los cuernos en perspectiva, trazos cortos verticales correspondientes a la giba del animal, la línea frontal y la oreja. La alteración de la pared se debe sobre todo a la deposición de arcilla mediante los dedos, al ser un punto de apoyo. Las dimensiones máximas son de 51 x $40 \mathrm{~cm}$. Está a 159 $\mathrm{cm}$ del suelo.

B.II.3- Línea sinuosa cóncavo-convexa, ligeramente separada de las anteriores grafías $(125 \mathrm{~cm}$ a la izquierda). Mide $31 \mathrm{~cm}$. Se sitúa a $180 \mathrm{~cm}$ del suelo.

B.II.4- Mancha roja situada entre el bisonte B.II.2 y la línea B.II.3. Mide 1,5 x 1,5 cm. Está a $171 \mathrm{~cm}$ del suelo.

\subsubsection{Sector $C$}

Al final del pasillo del sector B, superando un desnivel ascendente de $3 \mathrm{~m}$ se accede a una nueva galería sinuosa y estrecha muy alterada por grafitis y con el suelo repleto de carbones procedentes de hogueras encendidas por los visitantes de fines del siglo XX. En

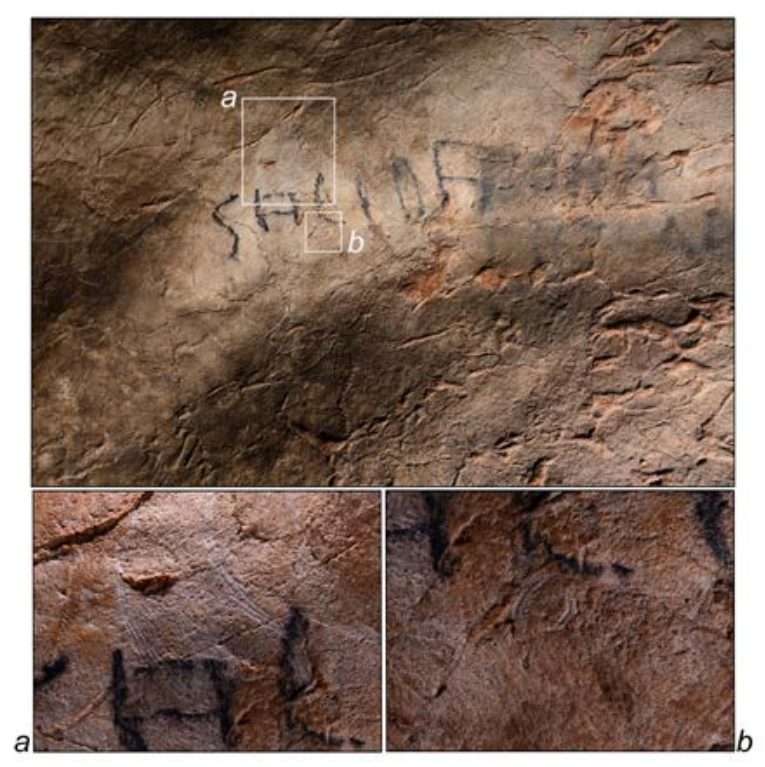

Fig. 6. Fotografía general y de detalle del bisonte B.I.3, grabado en el sector B de Aitzbitarte V (O. Rivero y D. Garate). En color en la edición electrónica.
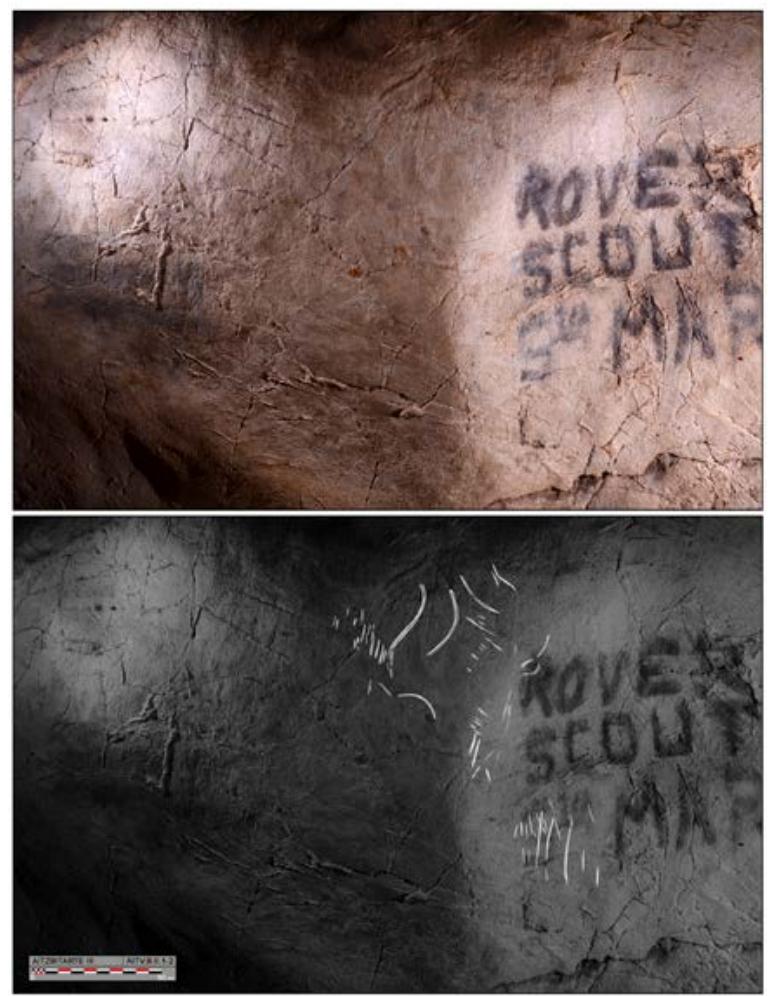

Fig. 7. Fotografía y calco del panel B.II con los bisontes B.II.1 y B.II.2, grabados en el sector B de Aitzbitarte V (O. Rivero y D. Garate). En color en la edición electrónica. 
la pared izquierda a medio recorrido de la misma, se grabó un bisonte (Fig. 8).

C.I.1- Parte superior de un bisonte orientado a la izquierda. Presenta un solo cuerno con doble línea, el arranque de la giba, la línea frontal y los pelos de la barba. Se reconoce también una pata delantera con pezuña y restos de la segunda. Está grabado con trazo único. Parte de la figura está destruida por grafitis modernos. Las dimensiones máximas son de $11 \times 9 \mathrm{~cm}$. Está a $168 \mathrm{~cm}$ del suelo.

\subsubsection{Sector D}

En el fondo de la cavidad se localiza la sala circular D de unos 4 × $5 \mathrm{~m}$, con varias oseras en el suelo. Se halla especialmente alterada por grafitis, hogueras y todo tipo de intervenciones vandálicas. Cuenta con cuatro paneles decorados. En el de la izquierda hay dos representaciones parciales de bisonte. El par de la derecha tiene una cabeza de animal y dos de bisonte respectivamente. El cuarto panel se sitúa en la pared derecha junto a la entrada y tiene líneas digitales.

En el panel izquierdo D, según se accede al sector, se observan dos bisontes parciales muy sumarios (Fig. 9).

D.I.1- Bisonte orientado a la izquierda y reducido a la parte superior. Presenta dos cuernos separados y sin perspectiva (perfil absoluto), uno de ellos continuado en la línea frontal y el otro prolongado para la ejecución de la giba y el lomo. Está grabado con trazo único. Las dimensiones máximas son de 66 x 30 $\mathrm{cm}$. Se sitúa a $123 \mathrm{~cm}$ del suelo.

D.I.2- Cabeza de bisonte orientada a la izquierda formada por la línea frontal unida a un cuerno, una serie de trazos desorganizados representando la testuz y otros más esporádicos para la barba. Las dimensiones máximas son de $15 \times 11 \mathrm{~m}$. Está a $116 \mathrm{~cm}$ de la repisa.

En la pared situada al fondo desde la entrada D.II, en el lado derecho y frente al panel siguiente, se observa una cabeza animal grabada (Fig. 10).

D.II.1- Cabeza de animal indeterminado orientado a la izquierda. Presenta una hilera de trazos largos, una línea a modo de frontal y otra inferior de trazos. Es probable que represente un équido. Se ha grabado con trazo único. Las dimensiones máximas son de $9 \times 18$ $\mathrm{cm}$. Se sitúa a $120 \mathrm{~cm}$ del suelo.

En el extremo derecho de la sala D.III se forma una pequeña hornacina o concavidad donde se identifican dos bisontes, uno con un solo cuerno y barba y el otro con los cuernos en perspectiva torcida y trazo múltiple (Fig. 10).

D.III.1- Bisonte orientado a la izquierda y reducido a la cabeza. Únicamente se observa un cuerno, la línea fronto-nasal y el hocico. Se ha grabado con tra-
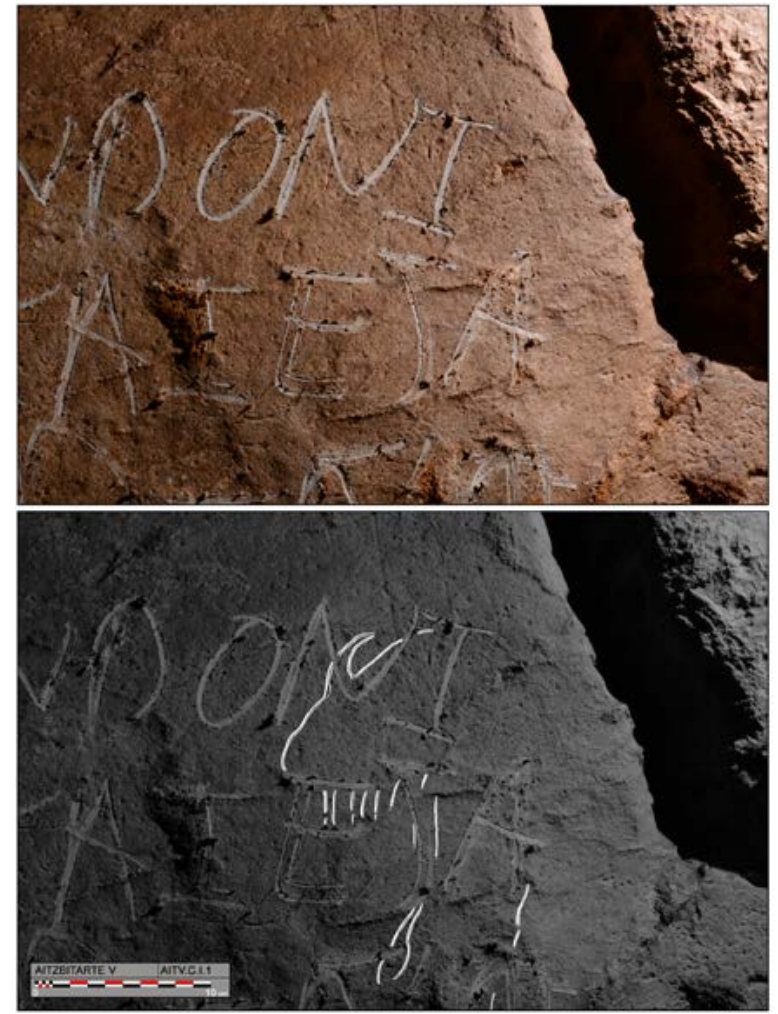

Fig. 8. Fotografía y calco del bisonte C.I.1, grabado en el sector C de Aitzbitarte V (O. Rivero y D. Garate). En color en la edición electrónica.

zo único muy fino. Las dimensiones máximas son de 14 x $6 \mathrm{~cm}$. Se alza a $135 \mathrm{~cm}$ del suelo.

D.III.2- Bisonte orientado a la izquierda y reducido a los cuernos sinuosos, ejecutados mediante trazo múltiple. Las dimensiones máximas son de 14 x $6 \mathrm{~cm}$. Se sitúa a $138 \mathrm{~cm}$ del suelo.

Por último, cerca del acceso al sector y todavía en la pared del lado, se ven una serie de trazos digitales fosilizados (Fig. 10):

D.IV.1- Serie de líneas sinuosas verticales fosilizadas y recubiertas por grafitis. Son cuatro trazos digitales de surco ancho y poco profundo que han desplazado la superficie limo-arcillosa original de la superficie de la pared. Las dimensiones máximas son de 13 x $14 \mathrm{~cm}$. Está a $105 \mathrm{~cm}$ del suelo.

\subsection{Valoración del conjunto}

La cueva de Aitzbitarte V presenta cuatro sectores decorados con representaciones mayoritariamente figurativas grabadas: 11 bisontes frente a 4 grupos de líneas o signos. Los cuatro sectores decorados están 

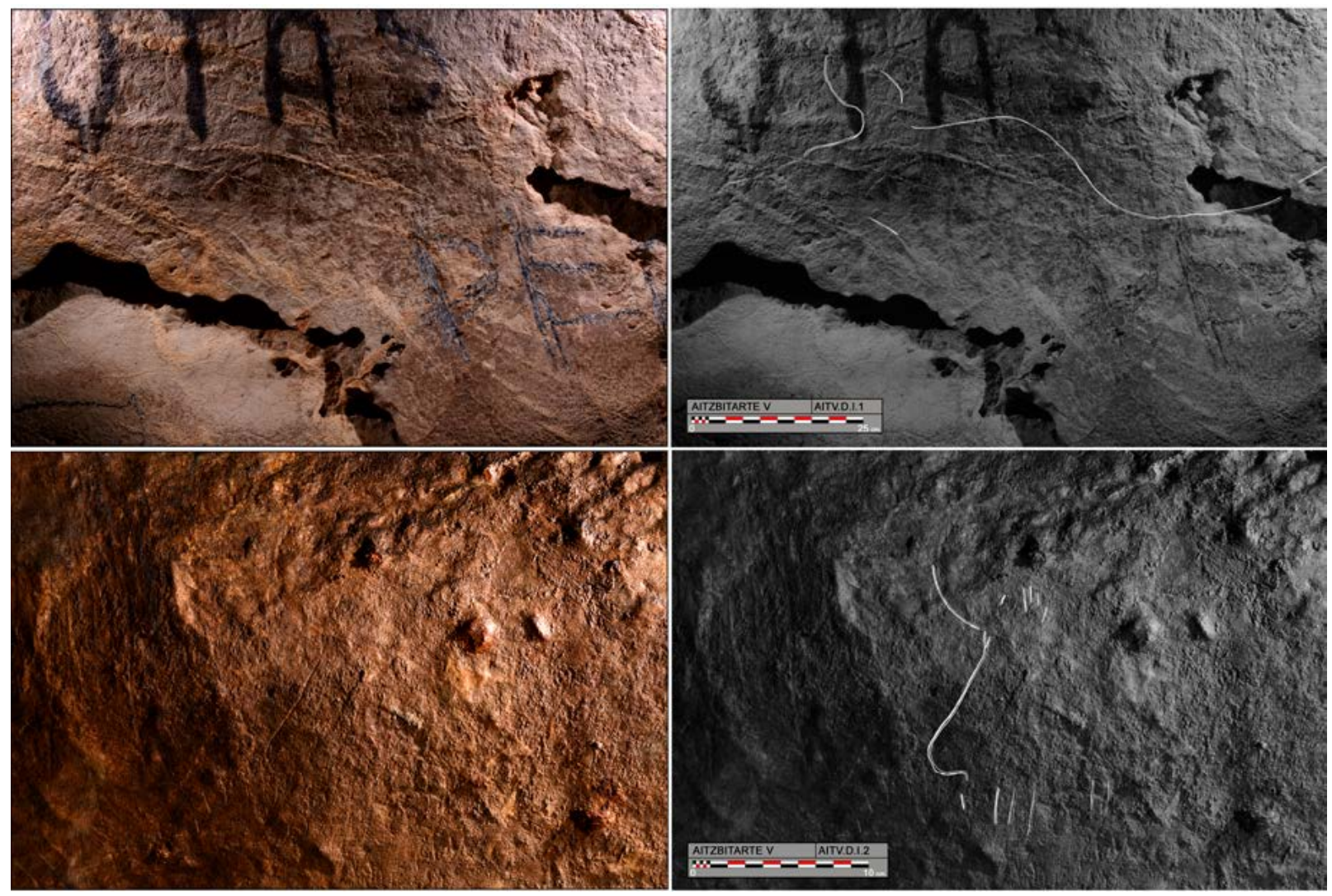

Fig. 9. Fotografía y calco de los bisontes D.I.1 y D.I.2, grabados en el sector D de Aitzbitarte V (O. Rivero y D. Garate). En color en la edición electrónica.

en espacios angostos, con accesos condicionados por pendientes y estrecheces. En los sectores A, B y C el aforo se limita a 2-3 personas como máximo. El D permite un aforo de unas seis personas (Fig. 11). Las intervenciones arqueológicas en la entrada no han documentado evidencias de asentamientos paleolíticos que sin embargo son muy intensos y continuados desde el Auriñaciense hasta el Aziliense en las cuevas de Aitzbitarte III y IV, cuyas bocas están situadas a escasos metros de la entrada de la V.

A pesar de compartir un mismo espacio topográfico, las representaciones descubiertas en el fondo de la cueva de Aitzbitarte V (Tab. I), poseen unas características muy heterogéneas desde el punto de vista técnico y formal. Ello permite enmarcarlas en dos momentos crono-culturales diferentes.

Técnicamente, excepto la mancha roja B.II.4, el grabado es el método empleado en esta cueva en variantes diferentes, probable consecuencia de la distinta dureza de los soportes calizos. En los sectores A, C y D, donde la superficie es blanda fruto de la alteración de la matriz caliza, el grabado es algo más profundo y una sola pasada con el útil grabador basta para marcar las líneas. En cambio, en el sector B, la superficie caliza de los soportes es de mayor dureza. Los surcos son menos profundos, indicando que fueron necesarias varias pasadas con el útil para marcar las líneas. En general, los grabados están relativamente bien conservados y sin pátina de alteración. Su color gris destaca sobre el fondo pardo-rojizo de la pared caliza.

Los prótomos de bisonte localizados en los sectores $\mathrm{A}, \mathrm{C}$ y $\mathrm{D}$ se caracterizan por su reducido tamaño: el bisonte A.II.01 no llega a los $10 \mathrm{~cm}$ de largo. Solo podrían verse desde distancias muy cortas. Su localización en lugares elevados y de acceso complejo dificultaría su visibilidad. Convenciones como los cuernos unidos a línea fronto-nasal/giba, su unión a base de trazos sueltos representando (A.I.1 y A.II.1) o no pelaje (D.I.1) aparecen también en las cuevas Aitzbitarte III y IX (Garate et al. 2016), en la recientemente descubierta Alkerdi 2 (Garate et al. 2017), así como en los soportes muebles decorados en la cercana cueva de Isturitz (Rivero y Garate 2014). De hecho, hasta los descubrimientos en las cuevas de Aitzbitarte, 


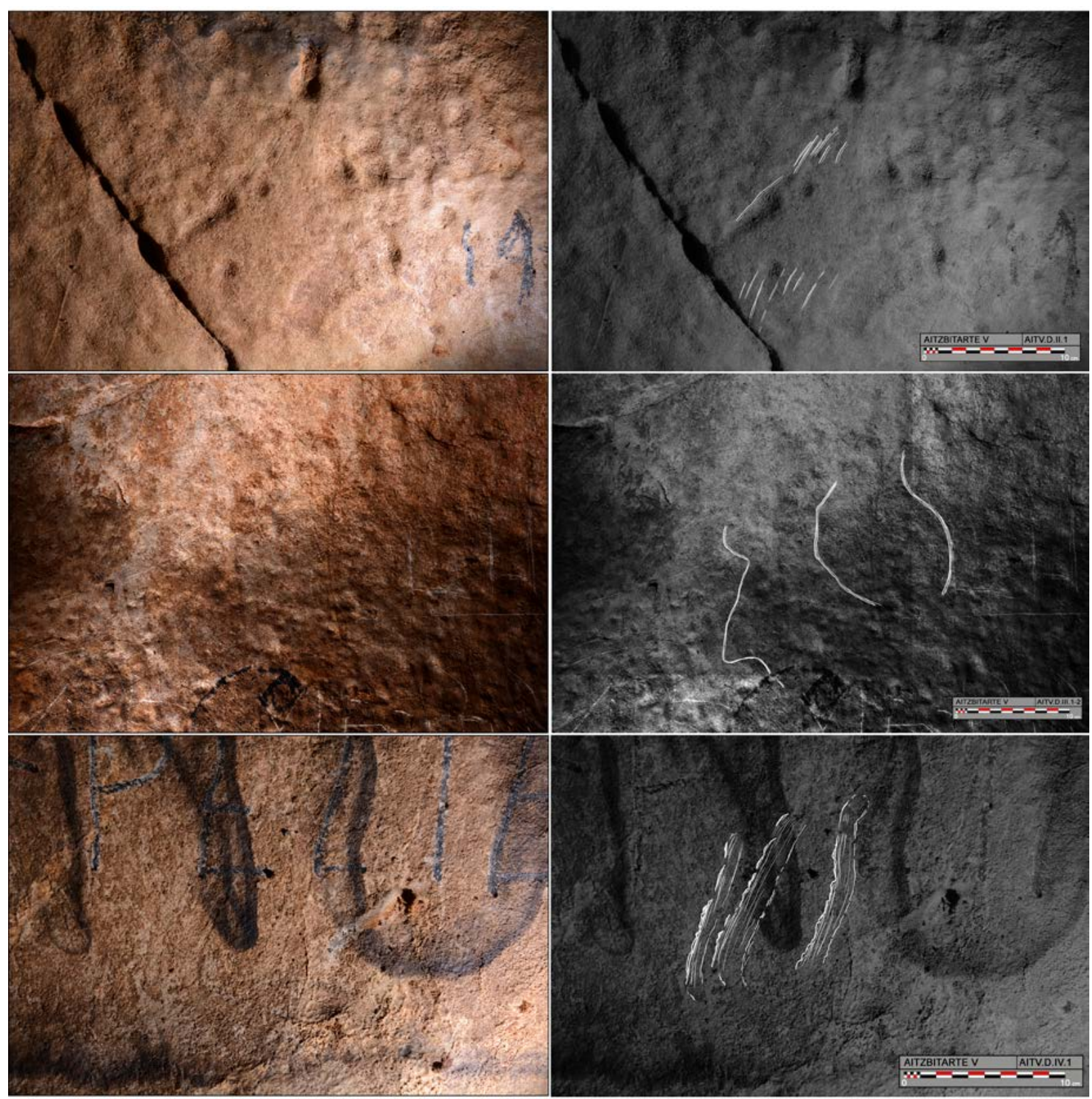

Fig. 10. Fotografía y calco del animal indeterminado D.II.1, de los bisontes D.III.1 y D.III.2 y de las líneas digitales D.IV,1, grabados en el sector D de Aitzbitarte V (O. Rivero y D. Garate). En color en la edición electrónica.

estas convenciones faltaban en la Región Cantábrica, siendo características de momentos gravetienses del S/ SO de Francia (cuevas de Gargas, Cussac, Cosquer) (Jaubert 2008).

El tamaño de las figuras localizadas en el Sector B es más común en las representaciones del Paleolítico Superior (media de un metro de largo) y suelen ubicarse a ambos lados de la estrecha galería final.
Además, se ven sin dificultad una vez traspasado el túnel que las separa de la parte anterior de la cueva. Sin embargo, el Sector B se halla al fondo de la cavidad y no es fácilmente accesible por el pequeño desnivel previo a la entrada que la separa del resto de la "Galería de los Osos". Los cuernos en perspectiva, la abundancia de detalles anatómicos, como el ojo en el bisonte B.I.3, la oreja en el bisonte B.II.2 


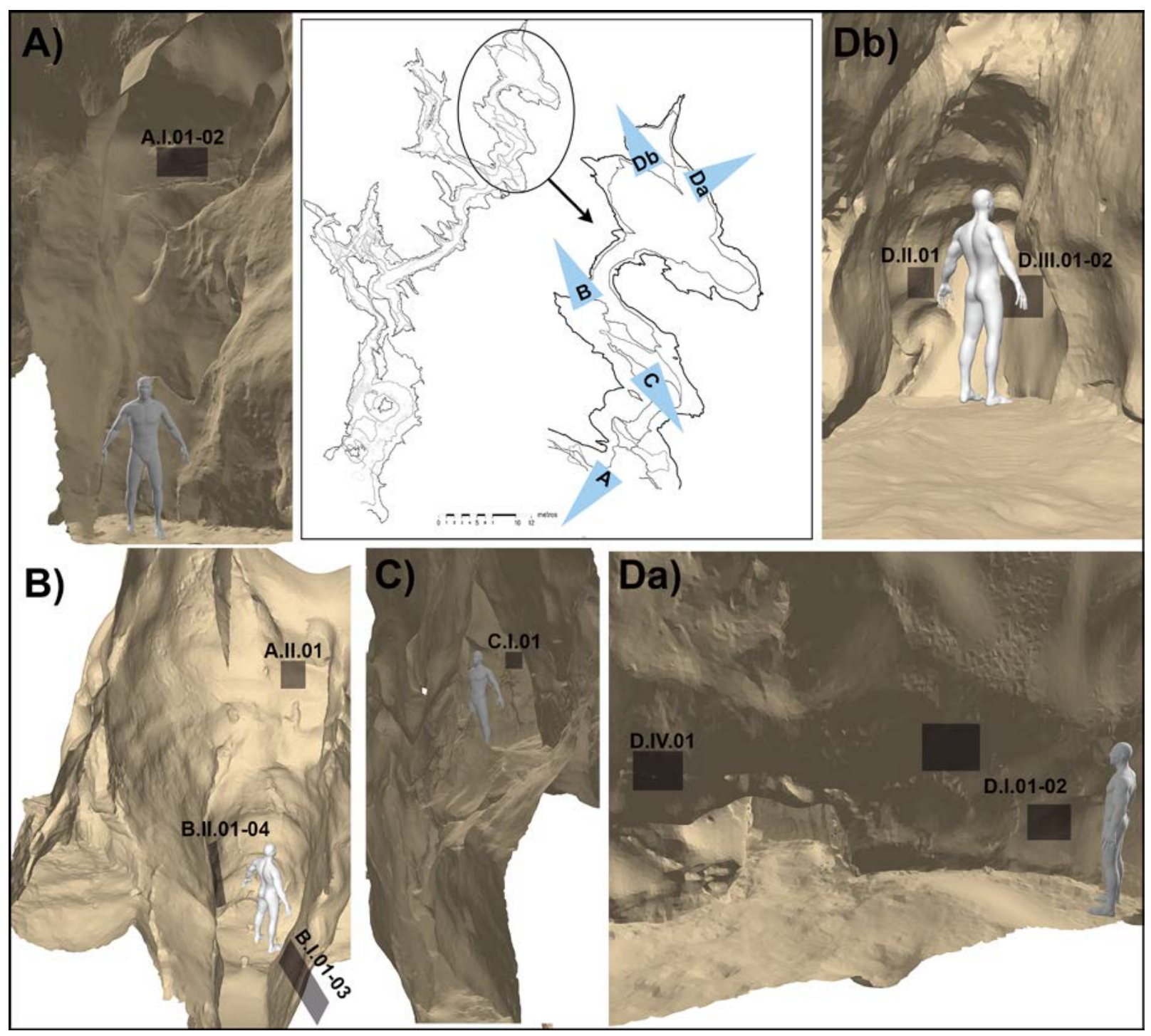

Fig. 11. Modelos tridimensionales de los tres espacios decorados en la cueva de Aitzbitarte V (I. Intxaurbe a partir de Gim-Geomatics, S.L.). A- sector A (vista hacia el panel A.I); B- sector B (vista hacia los paneles B.I y B.II); C- sector C (vista hacia el panel C.I); Da- sector D (paneles D.I y D.IV); Db- sector D (paneles D.II y D.III). Los recuadros oscuros indican la posición de los paneles decorados, los triángulos azules la orientación del observador, desde el lado menor de cada uno de ellos. En color en la edición electrónica.

y la representación del pelaje mediante trazos cortos paralelos en la giba y la barba, los vinculan a los bisontes del Magdaleniense reciente y, en particular, al conocido como "morfotipo Niaux" del contexto pirenaico (Fortea et al. 2004; Sauvet y Rivero 2016). Los paralelismos más cercanos en la ejecución de la línea fronto-nasal mediante series de trazos cortos paralelos de los bisontes están en la "Galería de los Bisontes" de la cueva de Alkerdi 1 (Garate y Rivero 2015), y en las cavidades pirenaicas de Marsoulas (Fritz y Tosello 2005), Trois-Frères (Bégouën y Breuil
1958), Fontanet (Vialou 1986) o Niaux (Clottes 1995). Es difícil atribuir el conjunto a una fase concreta del Magdaleniense, puesto que las convenciones de representación de los bisontes en la región pirenáica son casi idénticas en las fases media y superior (Sauvet y Rivero 2016). Sin embargo, su comparación con el arte mueble es muy clarificadora. La convención de definir la línea fronto-nasal únicamente mediante series de trazos cortos - muy rara y escasa en las representaciones muebles y parietales- es exclusiva del arte mueble del Magdaleniense Medio y se 
conoce en yacimientos tales como Las Caldas, Isturitz, Bedeilhac, Labastide, Mas d'Azil y Puy de Lacan (Rivero 2010).

Es decir, el arte parietal paleolítico de la cueva de Aitzbitarte V, tanto en su fase gravetiense como en su fase magdaleniense, tiene mayor afinidad con los contextos transpirenaicos que con los cantábricos (Fig. 12). En general, esta vinculación con el mundo pirenaico se constata también en otros aspectos de las ocupaciones paleolíticas de Aitzbitarte como las características de las industrias líticas y óseas. Seguramente está motivada por su cercanía al paso occidental de los Pirineos. En particular, como sucede con las cuevas localizadas en el núcleo de Alkerdi-Berroberria, las del cerro de Aitzbitarte pudieron haber funcionado como yacimientos "satélites" del núcleo de la colina de Gaztelu -cuevas de Isturitz, Oxocelhaya y Erberua-, por las estrechas relaciones que se observan tanto a nivel de las ocupaciones paleolíticas (Altuna et al. 2011) como en el arte parietal gravetiense y magdaleniense.

\section{DISCUSIÓN: LA RECURRENCIA GRÁFICA DURANTE EL PALEOLÍTICO SUPERIOR}

La presencia de palimpsestos gráficos en el arte rupestre se observó desde el comienzo de la investigación (p. ej., Alcalde del Río et al. 1911). Sin embargo, el uso recurrente de cuevas con fines simbólicos durante diferentes periodos es un tema sobre el que se ha investigado principalmente a partir de la incorporación de los análisis directos por C14-AMS (Valladas et al. 2013). Se ha planteado la presencia de distintas fases de ejecución a partir de los resultados de las dataciones de radiocarbono en cuevas decoradas francesas como Cougnac (Lot) (Lorblanchet 1994), Cosquer (Bouches du Rhône) (Valladas et al. 2017), las pinturas del Salon Noir en Niaux (Ariège) (Clottes et al. 1992) o incluso en El Castillo (Cantabria, España), donde se ha propuesto la existencia de figuras retocadas en distintas épocas a partir de las dataciones de radiocarbono (Valladas et al. 2001). Sin embargo, la validez de la mayoría de estas dataciones ha sido criticada por los posibles procesos de contaminación durante el muestreo y el tratamiento previo, que habrían podido rejuvenecer significativamente algunos de los resultados (Sauvet 2004). Por otro lado, las superposiciones estratigráficas en paneles principales de varias cuevas han sugerido una recurrencia espacial de la actividad simbólica. En la Región Cantábrica, este fenómeno se observó especialmente en las cuevas de Altamira y El Castillo, desde los primeros momentos de investigación (Alcalde del Río et al. 1911). Mas tarde se han añadido las cuevas de Peña Candamo,
Llonín, Tito Bustillo, La Pasiega y La Garma, cuyas superposiciones sugieren periodos de uso muy dilatados (González-Sainz 2004; González-Sainz y RuizRedondo 2010). A la vez se ha propuesto que hubiera un fenómeno de apropiación gráfica para las cuevas de Llonín, Tito Bustillo, Lloseta y Buxu en la Región Cantábrica occidental (Fortea 2007), según el cual, durante el Magdaleniense, se decorarían paneles que ya lo fueron durante el Paleolítico Superior Inicial. Las figuras "nuevas" se superpondrían a las más "antiguas", creando así palimpsestos complejos. Este mismo modelo parece aplicarse también en las cuevas de Altamira, La Pasiega, El Castillo y La Garma de la Región Cantábrica central (Corchón et al. 2012).

De hecho, algunas de estas cuevas fueron ocupadas durante varios períodos del Paleolítico Superior. Por esta razón se interpretaron como "sitios de agregación" aquellos yacimientos donde el registro arqueológico es extremadamente rico durante uno o varios períodos como la cueva de Altamira (Cantabria, España) (Conkey 1980, 1992) y también como "super sitios" algunas cuevas pirenaicas francesas como Isturitz (Pyrénées-Atlantiques) o Mas d'Azil (Ariège) (Bahn 1982). Esta idea se ha desarrollado en la Región Cantábrica a través de las geografías sociales de los grupos paleolíticos (Moure 1994; Utrilla 1994; Utrilla y Martínez-Bea 2008; Rasilla y Duarte 2018), reinterpretándose, p. ej. Isturitz (Rivero 2014), como centro de producción de objetos decorados durante el Magdaleniense.

Recientemente, durante nuestra de investigación en el Cantábrico oriental y el Pirineo occidental, hemos identificado tres sitios donde se documenta esta recurrencia gráfica durante el Paleolítico Superior: Aitzbitarte IV y Aitzbitarte V, inéditos, y Erberua, ya conocido y reinterpretado en este sentido (Garate et al. 2020a).

El fenómeno de recurrencia gráfica parietal parece especialmente relevante en la Región Cantábrica (Fig. 13). De hecho, para el Magdaleniense Medio y Superior de un total de 40 cuevas decoradas, 14 presentan fases previas. Es decir, menos de $2 / 3$ son decoradas ex novo en la región durante este período. Sin embargo, las cuevas redecoradas son marginales en las demás regiones europeas con arte parietal paleolítico (Garate et al. 2020a). Además, hay una polarización muy marcada entre los sectores central/occidental y oriental, separados por un vacío de $200 \mathrm{~km}$, entre las cuencas del Asón y Oka, casi sin arte parietal magdaleniense. En el sector central/occidental, el porcentaje de cuevas reutilizadas se eleva al $41 \%$, entre las cuencas de los ríos Nalón y Asón. En el oriental, entre las cuencas de Asón y Bidasoa, solo podemos certificar la reutilización simbólica del espacio subterráneo en Aitzbitarte V -tal vez también en Aitzbitarte IV-, mientras que las 9 restantes se atribuyen únicamente a momentos avanzados del Magdaleniense. 


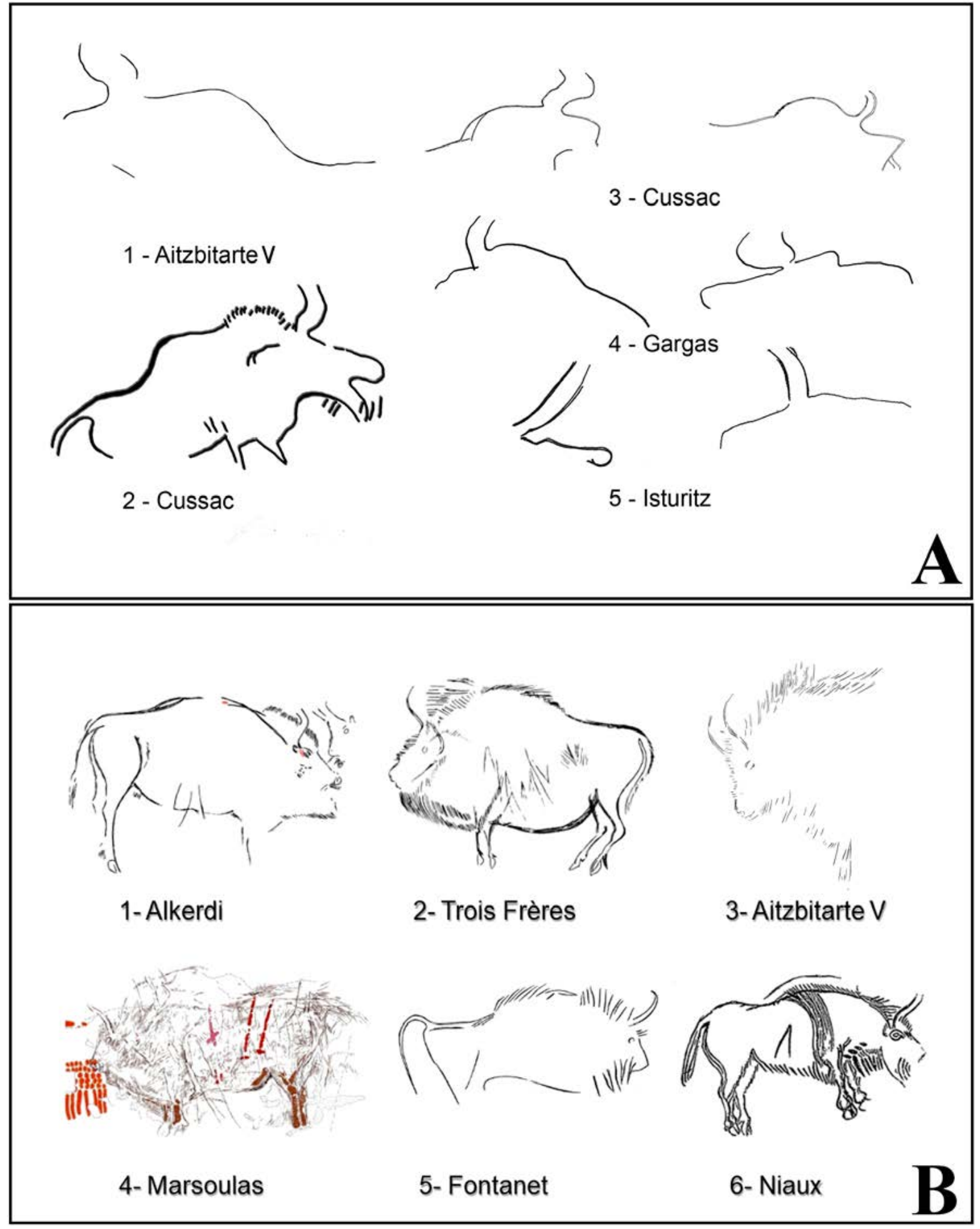

Fig. 12. A. Bisontes grabados gravetienses de estilo "Gargas" (2, 4 según N. Aujoulat et al. 2001; 3 según C. Barrière 1976; 5 según O. Rivero y D. Garate 2014). B. Bisontes grabados magdalenienses de estilo "pirenaico" (1 según D. Garate y O. Rivero 2015; 2 según H. Bégouën y Breuil 1958; 4 según C. Fritz y G. Tosello, 2005; 5 según D. Vialou 1986; 6 según J. Clottes 1995). En color en la edición electrónica. 
Al mismo tiempo, se distinguen tipos de reutilización en relación con la distribución espacial de las fases de decoración en el interior de las cuevas (Garate et al. 2020a). En el centro/oeste las fases antiguas del grupo de cuevas de Peña Candamo, Llonín, Tito Bustillo, Altamira y El Castillo se documentan por la mayoría de sus unidades topográficas. En cambio las recientes tienden a concentrarse en paneles principales, donde varias fases se superponen. En Pasiega A/B, Pasiega C/D y La Garma, El Buxu y Hornos de la Peña se dan esas superposiciones pero sin un espacio preferente. Un tercer modelo de distribución, identificado en El Salitre, Chufín, Aitzbitarte IV y Aitzbitarte $\mathrm{V}$, se caracteriza por la misma falta de estratigrafía parietal entre las diferentes fases que en la mayoría de los conjuntos pirenaicos con recurrencia gráfica $(\mathrm{Ga}-$ rate et al. 2020a). Es decir, el arte rupestre magdaleniense ocupa los espacios de la cueva que los artistas previos no seleccionaron.

\section{CONCLUSIÓN}

La cueva de Aitzbitarte V presenta dos fases decorativas bien diferenciadas por la presencia de convencionalismos específicos del arte gravetiense continen- tal transpirenaico y del magdaleniense pirenaico. Los conjuntos gráficos de ambas son modestos, compuestos por una docena de unidades gráficas figurativas situadas al fondo de una cavidad de reducidas dimensiones. La diferenciación de dos fases decorativas en una misma cueva resulta toda una novedad para la Región Cantábrica oriental, donde no se conocía dicho fenómeno hasta el momento. En todo caso, la distribución espacial segregada de ambos conjuntos dista del modelo cantábrico de palimpsesto y se aproxima más a cuevas pirenaicas como Erberua o Trois-Frères: fases gravetiense y magdaleniense medio con solapamiento espacial marginal o nulo.

Sin duda, la cueva de Aitzbitarte V aporta importantes novedades al escaso registro gráfico conocido en el oriente de la Región Cantábrica hasta los descubrimientos protagonizados en las dos últimas décadas en Atxurra, Armintxe o Aitzbitarte IV (Garate 2018). En esta ocasión, las novedades también parecen indicar una fuerte influencia pirenaica en el área geográfica estudiada en distintos periodos del Paleolítico Superior, en lo que al comportamiento gráfico se refiere. Queda pendiente evaluar si esa misma realidad se repite en todo el registro arqueológico y en todas las fases, o si por el contrario es un fenómeno reducido a ciertas manifestaciones arqueológicas o sólo

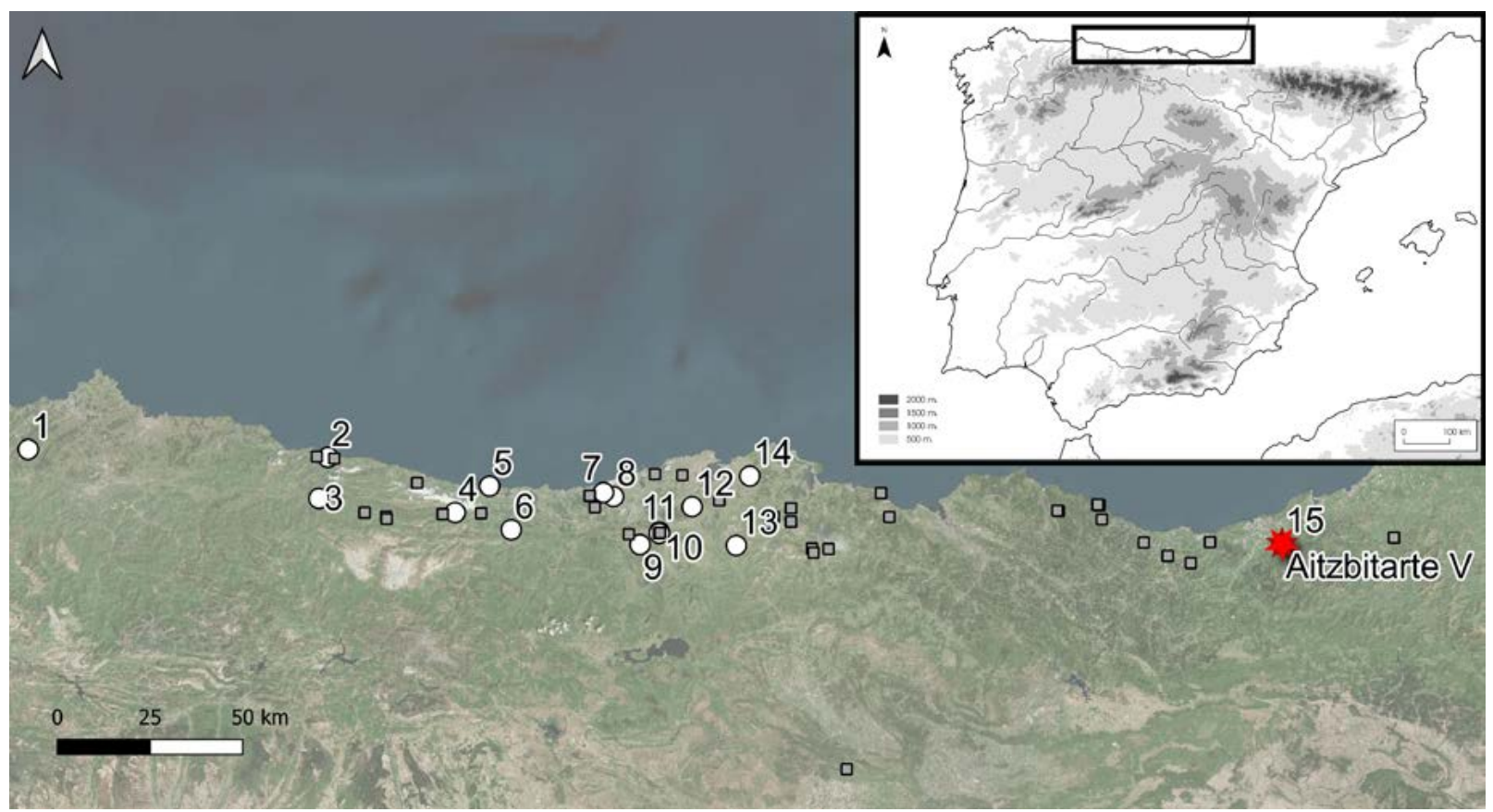

Fig. 13. Cuevas con recurrencia gráfica (círculos grandes en blanco) y cuevas decoradas ex novo (cuadrados pequeños grises) en la Cornisa

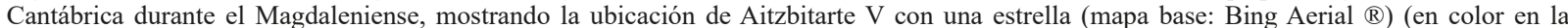
edición electrónica). 1: La Peña de Candamo, 2: Tito Bustillo, 3: El Buxu, 4: Llonín; 5: El Pindal, 6: Chufín, 7: Cualventi, 8: Altamira, 9: Hornos de La Peña, 10: La Pasiega, 11: El Castillo, 12: La Llosa, 13: El Salitre, 14: La Garma, 15: Aitzbitarte IV. 
evidente en algunos periodos concretos, como por ejemplo el gravetiense noaillense (Garate et al. 2020b).

\section{AGRADECIMIENTOS}

Los autores agradecen al grupo de espeleología "Felix Ugarte Elkartea" su intervención activa y crucial en los hallazgos y en las prospecciones, especialmente a J. Busselo, S. Laburu y A. Rodríguez.

\section{BIBLIOGRAFÍA}

Alcalde del Río, H.; Breuil, H. y Sierra, L. 1911: Les cavernes de la región cantabrique. Chêne. Mónaco.

Altuna, J. 2004: "Las cuevas de Aitzbitarte (Landarbaso, Renteria) (Historia de las investigaciones)". Kobie (serie anejos) 6 (1): 133-138.

Altuna, J.; Armendariz, A.; Etxeberria, F.; Mariezkurrena, K.; Peñalver, X. y Zumalabe, F. 1995: Carta Arqueológica de Gipuzkoa. II. Cuevas. Munibe (Antropologia-Arkeologia), Suplemento 10. Sociedad de Ciencias Aranzadi. Donostia-San Sebastián.

Altuna, J. y Mariezkurrena, K. 2011: "Estudio de los macromamíferos del yacimiento de Aitzbitarte III (Excavación de la entrada)". En J. Altuna, K. Mariezkurrena y J. Rios-Garaizar (eds.): Ocupaciones humanas en la Cueva de Aitzbitarte III (Renteria, País Vasco) sector entrada: 33.000-18.000 BP. Eusko Jaurlaritzaren Argitalpen Zerbitzu Nagusia. Vitoria-Gasteiz: 395-480.

Altuna, J. y Mariezkurrena, K. 2017: "Estudio de los macromamíferos de la cueva de Aitzbitarte III (Zona del Interior)". En J. Altuna, K. Mariezkurrena, J. Rios-Garaizar y A. San Emeterio Gómez (eds.): Aitzbitarte III (País Vasco). 26.000-13.000 BP (zona profunda de la cueva). Eusko Jaurlaritzaren Argitalpen Zerbitzu Nagusia. VitoriaGasteiz: 247-315.

Altuna, J.; Mariezkurrena, K. y Rios-Garaizar, J. 2011: Ocupaciones humanas en Aitzbitarte (País Vasco), 33.600-18.400 BP (zona de entrada de la cueva). Colección de patrimonio cultural vasco, EKOB 5 , Servicio Central de Publicaciones del Gobierno Vasco. Vitoria.

Altuna, J.; Mariezkurrena, K.; Rios-Garaizar, J. y San Emeterio, A. 2017: Ocupaciones humanas en Aitzbitarte III (País Vasco), 26.000-13.000 $B P$ (Zona profunda de la cueva). Colección de patrimonio cultural vasco, EKOB 8, Servicio Central de Publicaciones del Gobierno Vasco. Vitoria.

Aujoulat, N.; Geneste, J.; Archambeau, C. y Delluc, M. 2001: "La grotte ornée de Cussac". Bulletin de la Société Historique et Archéologique du Périgord 128 (3): 543-552.

Baca, M.; Popović, D.; Stefaniak, K.; Marciszak, A.; Urbanowski, M.; Nadachowski, A. y Mackiewicz, P. 2016: "Retreat and extinction of the Late Pleistocene cave bear (Ursus spelaeus sensu lato)". The Science of Nature 103 (92): 1-17, doi: 10.1007/s00114-016-1414-8.

Bahn, P. 1982: "Intersite and Inter-regional links during the Upper Palaeolithic: the Pyrenean evidence". Oxford Journal of Archaeology 1 (3): 247-288.

Barandiarán, J. M.; Altuna, J. y Elosegui, R. 1965: "Excavaciones en Aitzbitarte IV. Campaña de 1964". Munibe 17: 21-37.

Barrière, C. 1976: L'art pariétal de la grotte de Gargas. British Archaeological Reports International Series 14, Mémoire de 1'Institut d'Art Préhistorique de Toulouse. Oxford.

Bégouën, H. y Breuil, H. 1958 : Les cavernes du Volp: Trois Frères, Tuc d'Audoubert. Arts et métiers graphiques. Ciudad.

Clottes, J. 1995: Les cavernes de Niaux: art préhistorique en Ariège. Éditions du Seuil. París.

Clottes, J.; Valladas, H.; Cachier, H. y Arnold, M. 1992: "Des dates pour Niaux et Gargas". Bulletin de la Société préhistorique française 89 (9): $270-274$.
Conkey, M. 1980: "The identification of prehistoric hunter-gatherer aggregation sites: the case of Altamira". Current Anthropology 21 (5): 609-630.

Conkey, M. 1992: "Les sites d'agrégation et la répartition de l'art mobilier, ou: Y a-t-il des sites d'agrégation magdaléniens?". En: Le Peuplement magdalénien, actes du colloque (Chancelade 1988). CTHS, París: 9-25.

Corchón, M. S.; Garate, D.; Hernando, C.; Ortega, P. y Rivero, O. 2012: "Vers un modèle décoratif pour la grotte de La Peña de Candamo (Asturies, Nord de l'Espagne) à la lumière des nouvelles découvertes". En J. Clottes (ed.): L'art pléistocène dans le monde / Pleistocene art of the world / Arte pleistoceno en el mundo. Actes du Congrès IFRAO (Tarascon-sur-Ariège 2010), Symposium "Art pléistocène en Europe". Préhistoire, art et sociétés: Bulletin de la Société Préhistorique de l'Ariège 65-66 (2010-2011): 123-143.

Feruglio, V.; Aujoulat, N. y Jaubert, J. 2011: "L'art pariétal gravettien, ce qu'il révèle de la société en complément de la culture matérielle". En N. Goutas, L. Klaric, D. Pesesse y P. Guillermin (ed.): À la recherche des identités gravettiennes: actualités, questionnements et perspectives, actes du colloque d'Aix-en-Provence, octobre 2008. Société préhistorique française (mémoire 53). París: 243-255.

Fortea Pérez, J. 2007: "Apuntes sobre el arte paleolítico del Oriente de Asturias". En S. Ríos, C. García de Castro, M. de la Rasilla y J. Fortea (eds.): Arte rupestre prehistórico del Oriente de Asturias. Consorcio para el Desarrollo Rural del Oriente de Asturias. Oviedo: 205-250.

Fortea Pérez, J.; Fritz, C.; Garcia, M. A.; Sanchidrián Tortí, J. L.; Sauvet, G. y Tosello, G. 2004: "L'art pariétal paléolithique à l'épreuve du style et du carbone-14. Union Internationale des Sciences Pré et Protohistoriques (U.I.S.P.P.) VIII e Commission: Paléolithique supérieur". Colloque de Liège 2003: La Spiritualité. ERAUL 106, Université de Liège. Lieja: 163-175.

Fortea Pérez, J.; Rasilla, M. de la y Rodríguez-Otero, V. 2004: "L'art pariétal et la séquence archéologique paléolithique de la grotte de Llonín (Peñamellera Alta, Asturias, Espagne)". Préhistoire, Art et Sociétes. Bulletin de la Société Préhistorique de l'Ariège 59: 7-29.

Fritz, C. y Tosello, G. 2005: "Entre Périgord et Cantabres: les magdaléniens de Marsoulas". Territoires, déplacements, mobilité, échanges durant la Préhistoire 126: 311-327.

Garate, D. 2018: "New insights into the study of Palaeolithic rock art: Dismantling the 'Basque Country Void'". Journal of Anthropological Research 74 (2): 168-200.

Garate, D.; Ríos-Garaizar, J.; Rivero, O. y Ugarte Elkartea, F. 2016: "Trois nouvelles grottes ornées à Aitzbitarte (Pays Basque)". International Newsletter of Rock Art 75: 1-5.

Garate, D.; Ríos-Garaizar, J.; Ruiz-Redondo, A. y Tapia, J. 2013: "Evidencias de arte parietal paleolítico en la cueva de Aitzbitarte IV (Errenteria, Gipuzkoa)". Munibe (Antropologia - Arkeologia) 64: $33-42$.

Garate, D. y Rivero, O. 2015: "La 'Galería de los Bisontes': un nuevo sector decorado en la Cueva de Alkerdi (Urdazubi/Urdax, Navarra)". Zephyrus 75: 17-39.

Garate, D.; Rivero, O.; Intxaurbe, I. y Díaz-González, L. en prensa: "Back to the wall: an approach to the re-appropriation of subterranean symbolic spaces during the Late Upper Palaeolithic in the Bay of Biscay watershed". Bulletin de la Société Préhistorique Française 2020.

Garate, D.; Rivero, O.; Rios-Garaizar, J.; Arriolabengoa, M.; Intxaurbe, I. y Salazar, S. 2020: "Redefining shared symbolic networks during the Gravettian in Western Europe: new data from the rock art findings in Aitzbitarte caves (Northern Spain)"'. PLOS ONE 15 (10): e 0240481. https://doi.org/10.1371/journal.pone.0240481.

Garate, D.; Tapia, J.; Rivero, O.; Álvarez, I.; Abendaño, V.; Aranburu, A.... y Agirre, J. 2017: "Alkerdi 2: a new Gravetian rock art cave in the Western Pyrenees". International Newsletter on Rock Art 80: $10-12$.

González-Sainz, C. 2004: "Arte parietal en la región cantábrica: centros y peculiaridades regionales". En M. A. Fano (ed.): Las sociedades del Paleolítico en la Región Cantábrica. Kobie (Paleoantropología), anejo 8. Bilbao: 403-424. 
González-Sainz, C. y Ruiz-Redondo, A. 2010: "La superposición entre figuras en el arte parietal paleolítico. Cambios temporales en la región cantábrica". Cuadernos de Arqueología de Navarra 18 (1): 41-61.

Hoffmann, D. L.; Standish, C. D.; García-Díez, M.; Pettit, P. B.; Milton, J. A.; Zilhão, J.... y Pike, A. W. G. 2018: "U-Th dating of carbonate crusts reveals Neandertal origin of Iberian cave art". Science 359: 912-915.

Jaubert, J. 2008: “L'،art' pariétal gravettien en France: éléments pour un bilan chronologique". Paléo 20: 439-474.

Lorblanchet, M. 1994: "Le mode d'utilisation des sanctuaires paléolithiques”. En J. A. Lasheras (ed.): Homenaje al Dr. Joaquín González Echegaray. Ministerio de Cultura, Museo y Centro de Investigación de Altamira, Monografía 17. Madrid: 235-251.

Manteca, J.; Muñoz, R.; Mutiloa, I.; Sansinenea, K.; y Santafosta, M. 1997: "Estudio espeleológico de Aitzbitarte". Munibe (Ciencias Naturales - Natur Zientziak) 49: 3-47.

Moure, A. 1994: "Arte paleolítico y geografías sociales. Asentamiento, movilidad y agregación en el final del Paleolítico cantábrico". En T. Chapa y M. Menéndez (eds.): Arte Paleolítico. Complutum 5, Editorial Complutense. Madrid: 313-342.

Rasilla, M. de la y Duarte, E. 2018: “¿Casualidad o estrategia? Las aguas termales y minero-medicinales en la configuración de los yacimientos con arte rupestre paleolítico de Asturias y su correlato cantábrico". Nailos 5: 17-41.

Rios-Garaizar, J. y Garate, D. 2014: “Actualisation de l'inventaire des pointes de type Isturitz de la région cantabrique”. Paléo 25: 233-246.

Rios-Garaizar, J.; Peña, P. de la y Maillo-Fernández, J. M. 2013: “El final del Auriñaciense y el comienzo del Gravetiense en la región cantábrica: una visión tecno-tipológica". En C. de las Heras, J. A. Lasheras, A. Arrizabalaga y M. de la Rasilla (eds.): Pensando el Gravetiense: nuevos datos para la Región Cantábrica en su contexto peninsular y pirenaico. Monografías del Museo Nacional y Centro de Investigación de Altamira 23, Ministerio de Educación, Cultura y Deporte. Madrid: 369-382.

Ríos-Núñez, F. 2017: "Estudio de la industria ósea de la cueva de Aitzbitarte III (Zona del Interior)". En J. Altuna, K. Mariezkurrena, J. Rios-Garaizar y A. San Emeterio Gómez (eds.): Aitzbitarte III (País Vasco). 26.000-13.000 BP (zona profunda de la cueva). Eusko Jaurlaritzaren Argitalpen Zerbitzu Nagusia. Vitoria-Gasteiz: 187-246.

Rivero, O. 2010: La movilidad de los grupos humanos en el Magdaleniense de la Región Cantábrica y los Pirineos. Una visión a través del arte. Tesis Doctoral, Universidad de Salamanca. http://hdl.handle.net/10366/108927
Rivero, O. 2014: "Vers une caractérisation du gisement magdalénien d'Isturitz (Pyrénées-Atlantiques) à travers sa production artistique". Bulletin de la Société Préhistorique Française 111 (2): 255-274.

Rivero, O. y Garate, D. 2014: "L'art mobilier gravettien de la grotte d'Isturitz (fouilles Saint-Perier): une collection redécouverte". Paléo. Révue d'Archéologie Préhistorique 25: 103-120.

Rivero, O.; Ruiz-López, J. F.; Intxaurbe, I.; Salazar, S. y Garate, D. 2019: "On the limits of 3D capture: A new method to approach the photogrammetric recording of palaeolithic thin incised engravings in Atxurra Cave (northern Spain)". Digital Applications in Archaeology and Cultural Heritage 14, https://doi.org/10.1016/j.daach.2019.e00106.

Sanchidrián, J. L y Medina-Alcaide, M. A. 2017: "La prospección arqueológica de las cuevas con arte paleolítico. Una propuesta metodológica". Kobie, Serie Anejo 16: 27-40.

Sauvet, G. 2004: "Langage préhistorique, langages de préhistoriens". En F. Audouze y N. Schlanger (ed.): Autour de Leroi-Gourhan: contexte et actualité d'André Leroi-Gourhan. Éd. ADPCA. Antibes: 249-270.

Sauvet, G. y Rivero, O. 2016: “D'un support à l'autre: l'art pariétal à la lumière de l'art mobilier". Paléo numéro special: 133-147.

Tarriño, A. 2011: "Procedencia de los sílex de la cueva de Aitzbitarte III (Rentería, Gipuzkoa)". En J. Altuna, K. Mariezkurrena y J. RiosGaraizar (eds.): Ocupaciones humanas en la cueva de Aitzbitarte III (Renteria, País Vasco) Sector Entrada: 33.000-18.000 BP. Eusko Jaurlaritzaren Argitalpen. Vitoria-Gasteiz: 353-373.

Utrilla, P. 1994: "Campamentos-base, cazaderos y santuarios. Algunos ejemplos del paleolítico peninsular”. En J. A. Lasheras (ed.): Homenaje al Dr. J. González Echegaray. Ministerio de Cultura, Museo y Centro de Investigación de Altamira, Monografía 17. Madrid: 97-113.

Utrilla, P. y Martínez-Bea, M. 2008: "Sanctuaires rupestres comme marqueurs d'identité territoriale: sites d'agrégation et animaux 'sacrés"'. Préhistoire, art et sociétés 63: 109-133.

Valladas, H.; Quiles, A.; Delque-Kolic, M.; Kaltnecker, E.; Moreau, C.; Pons-Branchu, E.... y Delestre, X. 2017: "Radiocarbon dating of the decorated Cosquer cave (France)". Radiocarbon 59 (2): 621-633.

Valladas, H.; Tisnérat-Laborde, N.; Cachier, H.; Arnold, M.; Bernaldo de Quirós, F.; Cabrera-Valdés, V.... y Moure-Romanillo, A. 2001: "Radiocarbon AMS dates for Paleolithic cave paintings". Radiocarbon 43 (2B): 977-986.

Valladas, H.; Tisnérat-Laborde, N.; Cachier, H.; Arnold, M.; Bernaldo de Quirós, F.; Cabrera-Valdés, V.... y Moure-Romanillo, A. 2011: "Radiocarbon AMS dates for Paleolithic cave paintings". Radiocarbon 43 (2B): 977-986.

Vialou, D. 1986: "L'art des grottes en Ariège magdalénienne". XXIIe Suppl. à Gallia Préhistoire, CNRS. París.

Trab. Prehist., 77, N. ${ }^{\circ}$ 2, julio-diciembre 2020, pp. 320-336, ISSN: 0082-5638

https://doi.org/10.3989/tp.2020.12259 